\title{
Алгоритм численного моделирования равновесных цен на рассредоточенном рынке олигопсонного типа
}

\author{
А.С. Маничева, Ю.А. Алябышева, В.С. Дронов, А.В. Жариков, О.Н. Половикова
}

Алтайский государственный университет (Барнаул, Россия)

\section{Algorithm for Numerical Simulation of Set of Equilibrium Prices in a Spatially Distributed Market of Oligopsony Type}

\author{
A.S. Manicheva, Yu.A. Alyabysheva, V.S. Dronov, A.V. Zharikov, O.N. Polovikova
}

Altai State University (Barnaul, Russia)

\begin{abstract}
Исследованию моделей ценовой конкуренции и способам численного достижения ценового равновесия посвящено большое количество работ как отечественных, так и зарубежных ученых, рассматривающих в основном рынки олигопольного типа. В статье представлен вариант экономико-математической модели мультиагентного рассредоточенного рынка олигопсонной структуры при условиях пространственной распределенности агентов рынка, однопродуктовости, однородности продукции, существования ограничений по объему производства продукции, полной информированности агентов рынка, линейности функций затрат и отсутствия посредников. Дан алгоритм численного моделирования параметров конфигурации множества равновесных цен на рынке рассматриваемой структуры, основанный на итеративной процедуре согласования цен. Применение алгоритма дает информацию для выбора равновесной цены, определяющей оптимальный уровень спроса и предложения продукции на всем рынке в процессе ценовой конкуренции между потребителями продукции. На основе представленной модели возможно как теоретическое, так и прикладное изучение механизма взаимодействия участников мультиагентного рассредоточенного рынка олигопольного и олигопсонного типа.
\end{abstract}

Ключевые слова: метод Монте-Карло, численное моделирование, рассредоточенный рынок, мультиагентность, олигопсония, равновесная цена.

DOI 10.14258/izvasu(2019)4-15

Введение. Исследованию моделей ценовой конкуренции и способам численного достижения ценового равновесия посвящено большое количество работ, например [1-12]. В данной статье рассматривается модель рынка олигопсонного типа. Олигопсония представляет собой тип рыночной структуры, при котором на рынке присутствует небольшое число поку-
A large number of works by both domestic and foreign scientists devoted to the study of models of price competition and ways of achieving numerical price equilibrium. Such works are mainly devoted to markets of oligopoly type. The article considers a variant of the economicmathematical model of a multiagent spatially distributed market of oligopsony structure under several conditions such as spatial distribution of market agents, one-product, product homogeneity, the existence of restrictions on the volume of production, full awareness of market agents, the linearity of cost functions, and the absence of intermediaries. An algorithm for numerical simulation of configuration parameters of the set of equilibrium prices in the market of the structure under consideration is presented, based on the iterative procedure of price negotiation. The application of the algorithm provides information for choosing an equilibrium price that determines the optimal level of demand and supply of products in the entire market in the process of price competition between consumers of products. Both theoretical and applied study of the mechanism of interaction of participants in a multiagent spatially distributed market of the oligopoly and oligopsony type is possible relying on the proposed model.

Key words: Monte-Carlo method, numerical simulation, spatially distributed market, multiagent, oligopsony, equilibrium price. пателей и большое количество продавцов. Примером могут служить рынки сельскохозяйственной продукции. Представлен новый алгоритм численного моделирования параметров конфигурации множества равновесных цен на рынке рассматриваемой структуры, основанный на итеративной процедуре согласования цен. 
Постановка задачи. Рассмотрим вариант экономико-математической модели рассредоточенного рынка олигопсонной структуры, на котором функционируют ограниченное число потребителей и значительно большее число производителей продукции. В качестве товара на рынке выступает продукция сельскохозяйственных товаропроизводителей, используемая в производственном процессе предприятия-потребителя. Аналогами такого тынка могут выступать рынок зерна, молока и т.п.

Особенностями рассматриваемого рынка являются однородность продукции одного вида; осуществление взаимодействия между агентами рынка - предприятиями-производителями (далее просто производителями) и предприятиями-потребителями (далее просто потребителями) - по сделкам купли-продажи без перепродажи продукции между производителями; включение затрат на транспортировку и сбыт продукции на производителей, т.е. принятие во внимание пространственной распределенности агентов рынка.

Базовая экономико-математическая модель рассредоточенного рынка представлена в работах [13-15]. Различные варианты взаимодействия участников рынка исследовались в работе [16]. Рассмотрим вариант модели на примере рынка типичной олигопсонной структуры при следующих условиях: 1) производители завершили этап производства продукции и рассматривают варианты ее реализации, при этом имеющиеся переходящие запасы продукции и издержки на ее хранение не учитываются; 2) объемы предлагаемого на продажу сырья равны совокупным производственным мощностям потребителей; 3) потребители приобретают весь объем предложенной продукции, диктуя цену закупки.

Пусть на рынке присутствует $J$ потребителей $(j=1, \ldots, J)$ и $I$ производителей некоторой продукции $(i=1, \ldots, I)$. Агенты рынка действуют рационально в условиях полной информированности, в качестве критерия выбора решений агентов рассматривается максимизация прибыли.

Целью потребителя является максимизация прибыли от приобретения, переработки и дальнейшей реализации сырья; задача потребителя ј имеет вид:

$$
\begin{aligned}
& F_{j}(c, x)=\left(d_{j}-c_{j}-z_{j}^{p}\right) X_{j}(c) \rightarrow \max _{c_{j} \in \mathbf{c}_{j}}, \\
& \mathbf{c}_{j}=\left\{c_{j} \in R_{+}: c_{j} \in\left[c_{j}, \overline{c_{j}}\right]\right\}, j=1, \ldots, J,
\end{aligned}
$$

где $d_{j}$ - доходность единицы переработанного сырья; $z_{j}^{p}$ - затраты на переработку одной единицы сырья; $c_{j} \in \mathbf{c}_{j}-$ закупочная цена переработчика $j$, опреде-

ленная на интервале $\left[c_{j}, \overline{c_{j}}\right] ; \underline{c_{j}}-$ минимальная цена закупки сырья, при которой поступающее предложение отлично от нуля; $c_{j}$ - максимальная цена, при которой переработчик получит не нулевую прибыль;
$X_{j}(c)$ - объем закупленного и переработанного сырья.

Целью производителя является максимизация прибыли при распределении продукции на рынке между потребителями; задача производителя $i$ имеет вид:

$$
\begin{aligned}
& f_{i}(c, x)=\sum_{j=1}^{J}\left(c_{j}-r_{i j}-z_{i}\right) x_{i j} \rightarrow \max _{x_{i} \in \mathbf{x}_{i}}, \\
& \mathbf{x}_{i}=\left\{x_{i} \in R_{+}^{J}: 0 \leq \sum_{i=1}^{I} x_{i j} \leq \overline{x_{i}}\right\}, i=1, \ldots, I,
\end{aligned}
$$

где $r_{i j}$ - издержки на транспортировку и сбыт единицы сырья; $z_{i}$ - себестоимость единицы произведенного сырья; $x_{i}=\left(x_{i j}\right)_{j=1}^{J}-$ вектор предложения про-

дукции $i$-го производителя потребителям; $x_{i}=\sum_{j=1}^{J} x_{i j}-$ объем предлагаемой на рынок продукции; $\overline{x_{i}}$ - производственный потенциал, зависящий от используемой агротехнологии, климатических условий и пр.

Выражение (1)-(2) представляет собой теоретико-игровую модель рассредоточенного мультиагентного рынка.

Равновесные (компромиссные) цены, устанавливаемые на рынке в процессе ценовой конкуренции между потребителями и определяющие оптимальный уровень спроса $X_{1}^{*}(c), \ldots, X_{J}^{*}(c)$ и предложения $x_{1}^{*}(c), \ldots, x_{I}^{*}(c)$, образуют множество следующего вида:

$$
\mathbb{C}=\left\{c \in R_{+}^{J}: c \in[\underline{c}, \bar{c}] ; X_{j}\left(c_{j}\right)=\sum_{i=1}^{I} x_{i}(c), j=1, \ldots, J\right\} .
$$

Таким образом, на рассредоточенном рынке цена варьируется на интервале $[\underline{c}, \bar{c}]$, где $\underline{c}=\min _{j=1, \ldots, J}\left\{c_{j}\right\}$, a $\bar{c}=\max _{j=1, \ldots, J}\left\{\bar{c}_{j}\right\}$. В работе [17] было показано, что равновесная цена в модели (1)-(2) не единственна, если выполнены условия: а) $\left.c^{*} \in[\underline{c}, \bar{c}] ; \sigma\right) \underline{c}<\bar{c}$;

в) $\sum_{i=1}^{I} \bar{x}_{i} \geq \sum_{j=1}^{J} X_{j}\left(\underline{c}_{j}, d_{j}\right)$.

Оптимальное поведение производителя $i, i=1, \ldots, I$, модели (1)-(2) описывается через текущий объем продукции $\Delta x_{i j}$ следующим образом:

$$
x_{i j}=\left\{\begin{array}{c}
0, c_{j}<\underline{c_{i j}}, \\
\Delta x_{i j}, c_{j} \geq \underline{c_{i j}},
\end{array}\right.
$$

где $c_{i j}-$ минимальная цена реализации продукции.

Множество потребителей $\{1, \ldots, J\}$, рассредоточенных в пространстве и представляющих интерес для производителя $i$, описывается так:

$$
G_{i}=\left\{g_{k} \in\{1, \ldots, J\}: c_{g_{(k-1)}}-r_{i g_{(k-1)}} \geq c_{g_{k}}-r_{i g_{k}} \geq \varphi_{i}\right\},
$$


где $\varphi_{i}$ - минимальный объем выручки за минусом издержек производства, ожидаемый производителем при реализации продукции; $k-$ приоритет производителя ( $k$-й предпочтительнее $(k+1)$-го). Потребители упорядочены по величине ожидаемой доходности (привлекательности), а множество $G_{i}$ не содержит индексов потребителей, при реализации продукции которым не достигается требуемый уровень рентабельности, т.е. не выполнено $c_{j}>c_{i j}$.

Соответственно, объем предложения на рынке $k$-го приоритета для производителя $i$ составит:

$$
\begin{aligned}
& k=1: x_{i g_{1}}=\left\{\begin{array}{c}
x_{i}, x_{i} \leq X_{g_{1}}\left(c_{g_{1}}\right), \\
X_{g_{1}}\left(c_{g_{1}}\right), x_{i}>X_{g_{1}}\left(c_{g_{1}}\right),
\end{array}\right. \\
& k=2, \ldots, K_{i}: x_{i g_{k}}=\left\{\begin{array}{l}
\Delta x_{i g_{(k-1)}}, \Delta x_{i g_{(k-1)}} \leq X_{g_{k}}\left(c_{g_{k}}\right), \\
X_{g_{k}}\left(c_{g_{k}}\right), \Delta x_{i g_{(k-1)}}>X_{g_{k}}\left(c_{g_{k}}\right),
\end{array}\right.
\end{aligned}
$$

где $K_{i}=M\left(G_{i}\right)$ - мощность множества $G_{i} ;$ $\Delta x_{i g_{(k-1)}}$ - остаток продукции, нереализованной

производителю более высокой приоритетности

$$
\left(\Delta x_{i g_{(k-1)}}=x_{i}-\sum_{\tau=1}^{k-1} x_{i g_{\tau}}\right) \text {, при этом } x_{i j}=0 \text {, если } j \notin G_{i} \text {. }
$$

Алгоритм численного моделирования множества равновесных цен

В практике экономических вычислений используется несколько методов определения равновесия на рынке, основанных на итеративной процедуре согласования цен. Если итеративный процесс в конечном счете достигает равновесия на рынке, то лежащий в основе динамический процесс считается устойчивым, а равновесная цена - устойчивой $[18$, с. 264]. Основываясь на этом принципе, реализуем численное моделирование параметров конфигурации множества равновесных цен $\mathbb{C}$.

Введем вспомогательный показатель $\Psi_{j}$ для оцен-

ки баланса спроса и предложения - степень насыщения спроса на рынке $j: \Psi_{j}=\frac{\sum_{i=1}^{I} x_{i j}\left(c_{j}, r_{i j}\right)}{X_{j}\left(c_{j}\right)}$.

Если $\Psi_{j}>1$, то у потребителя $j$ в избытке пред-

ложение и для удовлетворения заданного уровня спроса цена может быть понижена; при $\Psi_{j}<1$ объ-

ем предложения недостаточен и цена возрастает; а при $\Psi_{j}=1$ достигается рыночное равновесие.

Пусть $l$ - номер итерации согласования цены в процессе торга.
При небольшом числе параметров компьютерной модели исследование свойств математических моделей эффективно проводить, как принято, например, в работе [19], на регулярной сетке. В нашем случае мультиагентного рынка для генерации начальных значений торговых цен $\left(c_{j}^{0}, j=1, \ldots, J\right)$ мы используем метод Монте-Карло, который позволяет получить представительную выборку экзогенных значений параметров в многомерном пространстве.

Корректировка цены в процессе рыночного торга на $l$-й итерации производится по правилу:

$$
c_{j}^{l}=\left\{\begin{array}{l}
\max \left\{c_{j}^{l-1}-\alpha_{j}, c_{j}\right\}, \Psi_{j}=1, \\
\min \left\{c_{j}^{l-1}+\beta_{j}, \overline{c_{j}}\right\}, \Psi_{j}<1,
\end{array}\right.
$$

где $\alpha_{j}, \beta_{j}$ - шаг изменения цены; $j=1, \ldots, J$.

Объем предложения продукции определяется на основе (3).

Алгоритм численного моделирования параметров конфигурации множества равновесных цен включает семь этапов и представлен на рисунке 1.

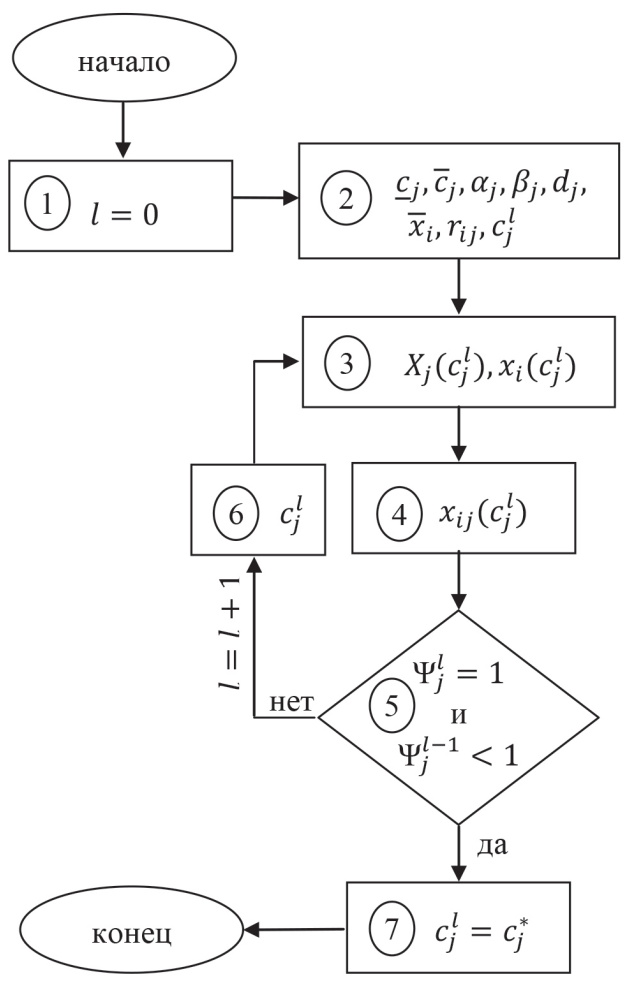

Рис. 1. Алгоритм численного моделирования

В результате реализации алгоритма получаем: величину $c_{j}^{*}$ - оптимальную равновесную цену потребителя $j ; x_{i j}^{*}$ - оптимальный объем реализации продукции (предложение) $i$-го производителя $j$-му потребителю с учетом степени его приоритетности; $X_{j}\left(c_{j}^{*}\right)$ - оптимальный спрос потребителя $j ; F_{j}\left(c_{j}^{*}\right)$ 
и $f_{i}\left(x_{i}^{*}\right)$ - максимальный объем прибыли участников рынка, полученной при рассматриваемом механизме их взаимодействия.

Численный пример. Рассмотрим апробацию алгоритма численного моделирования параметров кон- фигурации множества равновесных цен при следующих условиях: $J=2, I=5, \alpha=0,5, \beta=0,001$, тестовые данные, характеризующие агентов рынка, приведены в таблицах 1-2.

Таблииа 1

Числовые характеристики потребителей

\begin{tabular}{|l|c|c|}
\hline \multirow{2}{*}{ Характеристика } & \multicolumn{2}{|c|}{ Потребители } \\
\cline { 2 - 3 } & 1 & 2 \\
\hline Цена реализации готовой продукции, тыс. руб./т & 7,55 & 7,37 \\
\hline Затраты на переработку сырья, тыс. руб./т & 1,50 & 1,40 \\
\hline Минимальная цена закупки, тыс. руб./т & 1,01 & 0,98 \\
\hline Максимальная цена закупки, тыс. руб./т & 4,12 & 3,85 \\
\hline
\end{tabular}

Таблица 2

Числовые характеристики производителей

\begin{tabular}{|l|c|c|c|c|c|c|}
\hline \multicolumn{2}{|c|}{ Характеристика } & \multicolumn{5}{|c|}{ Производители } \\
\cline { 3 - 8 } & & 1 & 2 & 3 & 4 & 5 \\
\hline Производственный потенциал, т & 8600 & 8050 & 7720 & 4640 & 7418 \\
\hline \multirow{2}{*}{ Затраты на реализацию продукции потребителям, тыс. руб./т } & 1 & 0,25 & 0,42 & 0,51 & 0,17 & 0,08 \\
\cline { 3 - 8 } & 2 & 0,51 & 0,425 & 0,425 & 0,34 & 0,17 \\
\hline
\end{tabular}

Варианты начальных цен $c(j \quad 1,2)$ генерируются выше ценового интервала $[\underline{c}, \bar{c}]$, внутри интервала и ниже интервала. Среднерыночная цена, независимо от выбора начальных цен, достигается на одном и том же уровне (рис. 2).

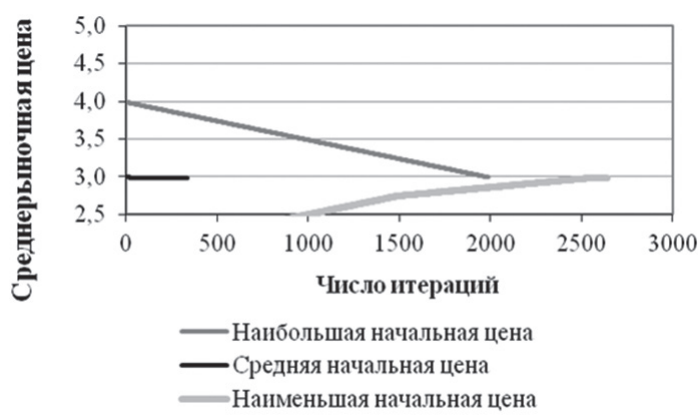

Рис. 2. Достижение среднерыночной цены при разных вариантах начальных цен

Элементы множества $\mathbb{C}$, отражающего варианты цен, обеспечивающих баланс спроса и предложения на рассредоточенном рынке, получены путем случайного многократного генерирования начальных торговых цен методом Монте-Карло и нахождением ближайшей равновесной цены $c^{*} \in \mathbb{C}$ (рис. 3 ).

Изучение множества $\mathbb{C}$ и его свойств позволит более подробно изучать механизм взаимодействия агентов рассредоточенного рынка.

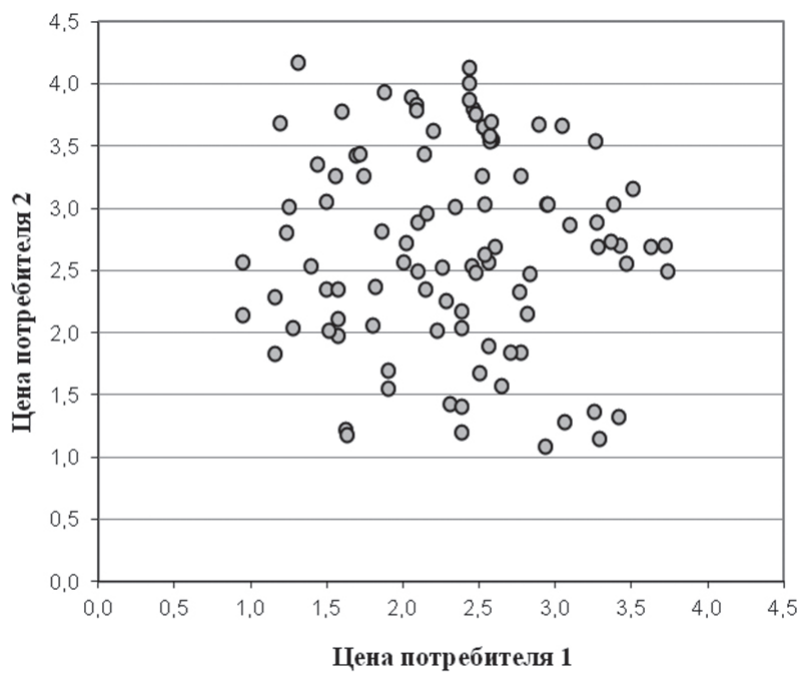

Рис. 3. Расчетные элементы множества компромиссных цен

Заключение. В статье рассмотрен вариант экономико-математической модели рассредоточенного рынка олигопсонной структуры, учитывающий пространственную рассредоточенность агентов рынка, ограничения по объему производства продукции и отсутствие посредников. Представленный алгоритм численного моделирования параметров конфигурации множества равновесных цен позволяет получить информацию для выбора оптимальной равновесной цены 
на рассредоточенном рынке в процессе ценовой конкуренции между потребителями, а также оптимальный объем реализации предложения продукции $i$-го производителя с учетом степени его деловой активности и оптимальный спрос потребителя продукции. На основе представленной модели возможно как теоретическое, так и прикладное изучение механизма взаимодействия участников мультиагентного рассредоточенного рынка олигопольного и олигопсонного типа.

\section{Библиографический список}

1. Булавский В.А., Калашников В.В. Метод однопараметрической прогонки для исследования состояния равновесия // Экономика и мат. методы. 1994. Т. 30. № 4.

2. Ruffin R.J. Cournot Oligopoly and Competitive Behaviour // Review of Economic Studies. 1971. Vol. 38. No. 4.

3. Okuguchi K. Quasi-Competitiveness and Cournot Oligopoly // The Review of Economic Studies. 1973. Vol. 40. No. 1. DOI:10.2307/2296748.

4. Sherali H.D., Soyster A.L., Murphy F.H. Stackelberg-NashCournot Equilibria: Characterizations and Computations // Operations Research. 1983. Vol. 31. No. 2.

5. Novshek W. On the Existence of Cournot Equilibrium // The Review of Economic Studies. 1985. Vol. 52. No. 1.

6. Thorlund-Petersen L. Iterative computation of Cournot equilibrium // Games and Economic Behavior. 1990. Vol. 2. No. 1. DOI:10.1016/0899-8256(90)90014-L.

7. Garg R., Kapoor S. Auction Algorithms for Market Equilibrium // Mathematics of Operations Research. 2004 Vol. 31. No. 4. DOI:10.1287/moor.1060.0216.

8. Tong J., Hu J., Hu J. Computing equilibrium prices for a capital asset pricing model with heterogeneous beliefs and margin-requirement constraints // European Journal of Operational Research. 2017. Vol. 256. No. 1. DOI:10.1016/j. ejor.2016.07.010.

9. Поддубный В.В. Оптимальная стабилизация рынка, описываемого модифицированной моделью ВальрасаМаршалла // Обработка данных и управление в сложных системах : сб. статей. Томск, 2004. Вып. 6.

10. Васин А.А., Васина П.А. Рынки и аукционы однородного товара. М., 2005.
11. Машунин Ю.К. Теория, математическое моделирование и прогнозирование развития рынка // Известия ДВФУ. Экономика и управление. 2016. № 4.

12. Коваленко А.Г. О поиске состояния равновесия пространственно рассредоточенных рынков несовершенной конкуренции однородного продукта // Экономика и мат. методы. 2018. Т. 54. № 1.

13. Понькина Е.В., Маничева А.С. Об одной модели рынка зерна // Известия Алт. гос. ун-та. 2009. № 1 (61).

14. Понькина Е.В., Маничева А.С. Имитационное моделирование рассредоточенного, мультиагентного рынка зерна // Вестник НГУ. Серия : Информационные технологии. 2010. Т. 8. № 2.

15. Маничева А.С. Математическое моделирование рассредоточенного рынка зерна в условиях олигопсонии // Известия Алт. гос. ун-та. 2013. № 1-1 (77). DOI:10.14258/ izvasu(2013)1.2-19.

16. Понькина Е.В., Захарова Ю.А. Модель рассредоточенного рынка при асимметрии распределения транспортных расходов между агентами // Известия Алт. гос. ун-та. 2013. № 1-2 (77). DOI:10.14258/izvasu(2013)1.2-18.

17. Понькина Е.В., Маничева А.С. Некоторые вопросы математического моделирования рассредоточенного рынка зерна // Известия Алт. гос. ун-та. 2011. № 1-1 (69).

18. Интрилигатор М. Математические методы оптимизации и экономическая теория. М., 2002.

19. Данько Е. В. Функция субъективной полезности инвестиционных решений в условиях информационной неопределенности и метод оценки ее параметров // Вестник НГУ. Серия : Информационные технологии. 2015. Т. 13. № 4. 